\title{
Perspectives on Telemedicine
}

\section{Ben-Jacob T ${ }^{1}$, Ben-Jacob $\mathrm{M}^{2 *}$ and Wang $\mathrm{D}^{2}$}

${ }^{1}$ Department of Medicine, Avalon University School of Medicine, USA

${ }^{2}$ Deapartment of Theoretical Mathematics, Mercy College, USA

\begin{abstract}
Telemedicine also denoted as telehealth and e-health, is the remote diagnosis and delivery of healthcare using telecommunications technology. This article presents a synopsis of some of the features associated with telemedicine including the training courses, the technology involved, the medical implications, and the underlying ethics.
\end{abstract}

Keywords: Telemedicine; Courses; Technology; Implementation; Medical implications; Ethics

\section{Introduction}

Telemedicine is medical care via the use of technology. It affords care to some who may not be in a position to see a doctor or a medical caregiver because they live remotely. The technology promotes medical care by allowing healthcare providers to share their diagnoses with other experts in the field. It is also used for recording and storing patient history and the transmission of scans such as x-rays and MRIs. Telemedicine has been touted as a mechanism to reduce outpatient visits and facilitate the filling of prescriptions (eVisit, 2017). With regard to patient satisfaction, statistics indicate that $75 \%$ are interested in having an evisit as opposed to an in-person visit with a physician. In addition, evisits increase the process by which patients move through a health care setting in the neighbourhood of $300 \%$. This could improve the safety and quality of care. Prescriptions can be filled over the Internet provided they comply with state regulations and the Drug Enforcement Agency (DEA), a federal agency (eVisit, 2017). This approach to medicine can lower the overall costs of healthcare in general. This paper will provide an overview of several essential aspects of telemedicine.

\section{Courses in Telemedicine}

There are onsite and online courses in telemedicine $[1,2]$. They tend to be relatively short in length as the assumption is that people taking the courses are well versed as medical professionals. One such program offers one-day training programs, both accredited by the American Telemedicine Association, in two different tracks, one in developing a program and the second one in telemedicine applications [1]. Other programs include seminars of several days or webinars. Given the critical [2] nature of the use of telemedicine when involved directly in the healthcare of individuals, our opinion is that these courses should be longer and cover more information. More practice and more case studies need to be integrated. We contend that these courses should encourage group work as opposed to just getting the participants familiar with the technology. When involved in a medical situation and the patient are geographically remote from the healthcare provider, teleconferencing for a consultation may be necessary. Advance practice with the technology and the art of consultation in tandem can be very important.

Telemedicine is a different venue from face-to-face medicine and one should not try to get telemedicine to mimic face-to-face medicine. The goal is to provide healthcare in both milieus, just in different modes. Assessment of the participants in telemedicine courses needs to be of the highest priority because of the potential seriousness of the consequences.
Initial assessment is the measure of the participants' beginning knowledge. It serves as a benchmark for information learned. Formative assessment is the measurement of progress made as the material is covered. Its goal is to provide ongoing feedback that can be used by instructors to improve teaching and by students to improve learning. Summative assessment measures the progress made at the end of the allotted time period allotted. It is used for evaluation purposes [3].

Assessment is also categorized by objective versus subjective. Objective assessment has one answer and subjective has more than one. Objective question types include true/false and multiple choice, etc. Subjective questions include open-ended questions and essays [3]. We maintain that it is the latter type that develops stronger preparation for the future ability of the participants.

One more way that assessment is classified is whether it is based on observation and discussion and considered informal, as opposed to formal, which is based on written evaluation. Formal assessment uses supporting data on which to base conclusions [4].

The goals are similar, if not the same for all types of assessment. Betterment or improvement of the learning is the ultimate target [5].

Educators of telemedicine should refer to Bloom's Taxonomy and focus on assessing the type of work that supports analysis, evaluation, and creativity.

Bloom's taxonomy is a categorization of educational goals. A subdivision of this triangular Figure 1 is as follows: Remember, Recognizing, Recalling, Understand, Interpreting, Exemplifying, Classifying, Summarizing, Inferring, Comparing, Explaining, Apply, Executing, Implementing, Analyse, Differentiating, Organizing, Attributing, Evaluate, Checking, Critiquing, Create, Generating, Planning and Producing.

Assessment design today often concentrates on the lowest level of the taxonomy-what do the participants remember. It is not linked to future performance. It does not measure a level of creativity. In telemedicine, educational assessment cannot be the application of rote procedure. Telemedicine deals with the human condition and each case is unique.

*Corresponding author: Marion Ben-Jacob, Ph.D., Department of Theoretical Mathematics, Mercy College, USA, Tel: 914-674-7524; E-mail: MBenJacob@mercy.edu

Received June 26, 2017; Accepted July 13, 2017; Published July 15, 2017

Citation: Ben-Jacob T, Ben-Jacob M, Wang D (2017) Perspectives on Telemedicine J Health Med Informat 8: 275. doi: 10.4172/2157-7420.1000275

Copyright: ( $) 2017$ Ben-Jacob T, et al. This is an open-access article distributed under the terms of the Creative Commons Attribution License, which permits unrestricted use, distribution, and reproduction in any medium, provided the original author and source are credited. 


\section{Bloom's Taxonomy}

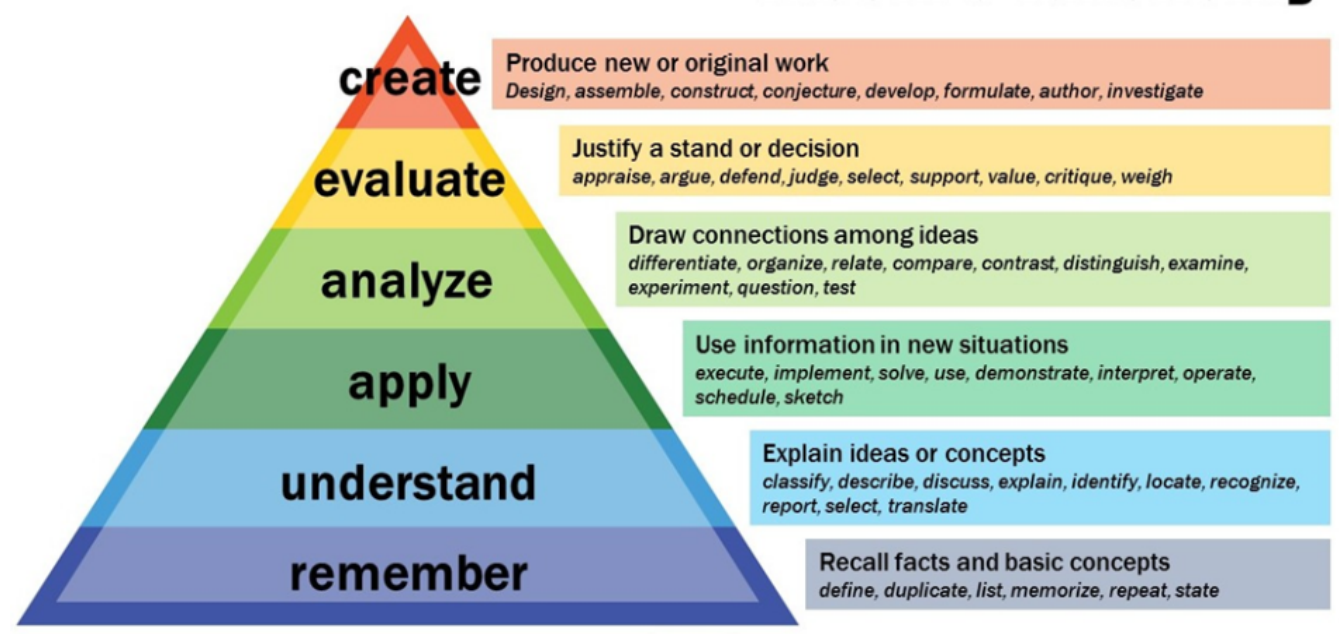

Figure 1: Bloom's Taxonomy is a classification of human cognition critical to the process of learning.

When we work at an innovative level, to create a new or original work, or to evaluate the work or design of someone else, we may opt to use ideas of our peers. We need to promote group work in a telemedicine course. The usual difficulty with a group project is who did what part and how much of the work. This can be overcome by asking each member of the group how they divided the work, piecewise, or collectively on each section of the project. In the latter case, each group member could be asked in confidentiality what percentage each member of the team contributed. In the professional world, teamwork is a given [6] consulting via teleconference for different opinions in a situation, if necessary, is a given in telemedicine.

The strategy for assessing individuals to prepare them for their future work is to give an individual assignment based on the concepts introduced in the course. This tactic will allow us to measure the progress of individual participants and realize the degree to which the group work aided him [3].

\section{Technology}

\section{Training courses}

Technology has been incorporated into the training of telemedicine be it via online learning or in an onsite course. Assessment is critical in the academic world. Technological tools have been developed to support evaluation techniques. One of the more popular ones is the use of clickers in a learning environment. This tool can be used is by giving each participant an individual clicker that has a specific ID connected to the participant's account. Multiple-choice responses to a question are afforded to all the participants who utilize their clickers to respond to the question posted by the instructor. There is a time limit for responses to be tallied and then the statistics associated with the responses, e.g. mean, mode, and median, become available. Individual scores are tabulated as well [7].

Blackboard, a popular platform for teaching online, also has tools built in for assessment. Participants can upload work that can be graded right away or held for future review. Blackboard allows for surveys, tests, quizzes, and electronic submission of assignments. A survey is a non-graded assessment tool that records answers anonymously with no individual results provided. This software allows for grading of discussion postings and instructor's comments, too [8].
The aforementioned tools are just a sampling of the electronic tools that might be used for teaching a course in telemedicine.

\section{Implementation of telemedicine}

On a simple level, one could use phones to relay symptoms and communicate with a professional. Of course, with today's technology, multiple platforms can be useful for telemedicine. There is Skype [9], which allows imaging as well as voice; platforms such as Blackboard are convenient for office hour discussions. There are teleconferencing systems such as WebEx [10] that provide on-demand collaboration, online meetings, web conferencing, and video conferencing. Video telephony supports the reception and transmission of audio-video displays for communication of users at different locations in real-time.

When medicine is involved, the communication needs to be more sophisticated to insure compliance with the United States law and confidentiality. Encryption is an algorithm for encoding a message so it can be read only by the sender and the proposed recipient, and no one who is unauthorized. Decryption is the reverse process of encryption to decode the encrypted data back to accessible data. Both encryption and decryption are required in telemedicine to safeguard data access. For greater detail, we will discuss encryption algorithms.

There are several algorithms utilized for encryption in telemedicine. They can be categorized by their speed, securitylevel, and implementation complexity/cost. Without going into technical depth, Galois Counter Mode/Advanced Encryption Standard are by far the most feasible and effective encryption method and yet, not every telemedicine system has incorporated it (Table 1). The governing law of computer encryption varies from country to country and even from state to state within the same country. So does the law on exporting and/or importing high-tech devices using encryption. These laws unavoidably impose challenges on the globalization of telemedicine. For example, when a U.S. telemedicine company exports its devices to China, it must not only comply with U.S. export regulations but also obey Chinese import and encryption regulations. Many nations do not have clear guidelines for import, export, or the standard of encryption. Even those countries that have clear guidelines often carry out inconsistent enforcement. Therefore, it is extremely essential to develop country-specific policies 


\begin{tabular}{|c|c|c|c|}
\hline & Single Churning & Triple Churning & Galois Counter Mode/Advanced Encryption Standard \\
\hline & Fast & Fast & Fast \\
\hline Speed & Low (14 bits key) & Low (48 bits key) & High (128 bits key) \\
\hline Implementation Complexity/Cost & Low & Low & High \\
\hline
\end{tabular}

Table 1: Ccomparisons of encryption algorithms in telemedicine.

that address the particular regulatory environment in each country, the telemedicine company's own needs, law compliance in that country, and the operating risks the company faces both technically and legally in that jurisdiction [11].

A typical telemedicine system consists of three essential components: an interface device for patients, an interface device for medical professionals, and a network connecting the former two components. When patients and medical professionals use a telemedicine system, authentications are the first step. For example, user login name, password, or other identification is required prior to the patient-doctor communication. The patient uses the interface to input data and/or scan the biological readings into the system. Telemedicine encrypts both the patient's identity and data/laboratory results and sends it over the network to a secure database. When the medical professional accesses the patient's data, the authentication process first has to validate his credentials; then the system grips data from the database and decrypts them into ready-to-access data. When the medical professional sends correspondence to the patient, the same encryption procedure is implemented. As we can realize, encryption acts as the security guard to hide data in telemedicine from unauthorized persons.

Security takes account of standards for the secure transmission of health information at the network level. The occurrences of interfering applications, e.g., spam, could delay the successful future of broadbandenabled telemedicine. Wi-Fi networks used at home tend to be less secure than wire-based ones, but because they are relatively inexpensive and can communicate with other wireless technologies, they are widely used. Popularity of telemedicine services and their impact on healthcare have stimulated private sectors to get involved in research in this area [12].

Second to security, network speed is another important factor that decides the expansion of telemedicine. Network speed, aka, bandwidth, is defined as the number of bytes of data $(\mathrm{B} / \mathrm{s})$ that can be transmitted from one point to another in one second within the network. Downloading means the user receives data and uploading means the user sends data. Video conferences, 3-D images, voices, and numerical charts are the typical telemedicine data, which make up huge volumes of pockets on a network; thus, they create network traffic. The slow network speed will hinder telemedicine future. Recent fibre deployments by major telecom corporations in the U.S. signal a dedication to providing end-users with faster, more reliable, and more versatile next-generation connections. Wireless carriers will leverage their advanced spectrum licenses and more effective spectrum management techniques to build the next generation networks [12]. Hopefully, with the speed of technological progress, these and other tools will soon are available for widespread use.

There are several best practices we can implement for telemedicine network infrastructure: availability, reliability, and maintainability [13]. Availability-what time range, what type of network, and which minimum bandwidth users can have to access the system. If the system is critical, the time range has to be 24/7/365. Local Area Network (LAN), Wide Area Network (WAN), Wi-Fi, and/or Virtual Private Network (VPN) can be the choices for network type depending on the cost and user location. Locations in rural areas are restricted to less bandwidth and therefore the system may use QoS (quality of service) software that gives priority to voice and video over other data packets traversing the network. In some instances, it is required to establish dedicated telehealth networks and to configure backup options should the primary network become unavailable [13]. Reliability-users can rely on all equipment when they use it. Hardware and software need to meet the industry standard especially for encryption. Maintainabilityjust like any device, telemedicine systems need to be maintained by either field professionals and/or users. The cost and the technical level of maintenance drive users' choice.

\section{Medical Implications}

Telemedicine generically means healthcare at a distance. Technology is used for many different aspects of healthcare. We present some examples here but our contention is that the greatest accomplishment is for technology to support the equivalent of routine healthcare on a widespread basis, especially for those who do not reside in the vicinity of good healthcare.

There are some applications of electronic consultations that are presently used for history review, ophthalmology determinations, and other purposes. Remote monitoring is used for managing specific conditions such as diabetes mellitus or enhanced joint management, among other conditions. Telemedicine is presently used in some emergencies but there are a number of caveats here. For example, providers are not always licensed and insurance companies are not willing to reimburse for the costs. In addition, physicians are not always willing to supply the necessary information to suit telemedicine applications for reimbursement either. Another concern is that a number of drugs that are used for chronic pain management cannot be prescribed without an in-person exam and so cannot even be prescribed via telemedicine platforms (eVisit, 2017).

Telepsychiatry, another aspect of telemedicine utilizes videoconferencing for patients residing in underserved areas to access psychiatric services. Teleradiology is the ability to send radiographic images, such as X-rays or CAT scans from one location to another. Telesurgery is the performance of surgical procedures where the surgeon is not physically in the same location as the patient, using a robotic system controlled by the surgeon. The remote operator may give tactile feedback to the user. Remote surgery combines elements of robotics and high-speed data connections [14].

Telemedicine patients are able to schedule appointments online or request an on-demand visit. Other benefits include the ability of providers to view their virtual waiting room and commence a virtual visit immediately, if necessary. There is an option for patients to receive a text message when the doctor is ready to see them. If more than one family member is being treated, family accounts are easily retrieved and managed [14].

Further management considerations include Health Information Technology (HIT). A health information management system supports the secure exchange of information between providers and patients [15]. The implementation of effective HIT will advance telemedicine services by providing ready access to critical health information a key component of HIT is the adoption of electronic health records (EHRs). 
As a part of the American Recovery and Reinvestment Act, all healthcare providers were required to implement meaningful use of EHRs by 2013. That is to say that EHRs need to achieve the following:

- Engage patients and their families;

- Improve care coordination, and population and public health;

- Maintain privacy and security of patient health information;

- Improve quality, safety, efficiency, and reduce health disparities.

As medical records are digitalized to EHRs, privacy, security, and authorized access are imbedded in telemedicine to ensure the system to meet both legal and technical requirements [16].

In countries that are emerging and not yet developed, telemedicine is essential; in remote areas, healthcare providers are at a minimum or lacking completely. The lack of a communication infrastructure needs to be overcome. There is often limited mobile connection, landline phones, or broadband connection. The best that can be hoped for here is the establishment of health centers that have power supplies. These centers are better for the local communities than no promise of healthcare at all.

\section{Ethics}

The ethical issues surrounding telemedicine are akin to those of face-to-face medical care. We seek a sound doctor- patient relationship, a protection of privacy, a treatment of diverse populations in an equitable manner and a practice of good healthcare [17].

Since the patient and the doctor are connecting via technology, the patient history may be compromised for no reason other than the patient is not completely comfortable with the technology used for electronic communication. This could potentially affect the quality of medical care [17].

Once again, the discrepancy in access to technology may affect an impartial treatment of different people based on their demographics and socioeconomic situation. Given some of the issues with technology today, e.g. hacking, patient privacy could be violated as well [17].

Another ethical issue is that of cross-border care. Since telemedicine involves patient and provider at a geographic distance, there is a potential for a conflict of laws. As an example, a practitioner who is licensed in a specific area treats a patient in another area where the practitioner is not licensed. The questions that arise include, did the practitioner violate the laws in the secondary area? What about liability if the patient is treated in a negligent manner [17]?

A United States law, the Health Insurance Portability and Accountability Act, better known as HIPAA, was developed by the Department of Health and Human Services to provided measures and benchmarks to protect patients' information that is provided to healthcare providers, organizations, and institutions. Electronic Protected Health Information, known as ePHI, is part of the 1996 HIPAA regulations. It speaks to all protected health information that is sent, received, or transmitted electronically. As such, the technological platforms that are used in telemedicine need to be completely encrypted and each healthcare provider needs to agree to a contract that they are HIPAA compliant [17].

By no means have we exhausted all the ethical issues with telemedicine. We just want to point out that, as with all innovation, there are pitfalls and issues to be resolved before society can totally benefit from the progress made.

\section{Conclusion}

Our contention is that all things being equal it is best to see a doctor in person to be examined; nevertheless, all things are not equal and there are extenuating circumstances. There were times when parents of new-borns could not get to a paediatrician's office. They relied on Dr. Benjamin Spock, a paediatrician who authored Baby and Child Care, published in 1946, touted as one of the best-sellers of all times, for guidance. Today, rather than rely on self-diagnosis with no or very limited medical training, telemedicine is a boon to society. As a concerned society, we need to make the technology available for those who need to use telemedicine for geographic or other sound reasons. Technology and medical advancements of the future hold promise that healthcare will be available on a widespread global basis.

\section{References}

1. http://telemedicine.arizona.edu/training.cfm

2. http://healthit.gov

3. Ben-Jacob M, Ben-Jacob T (2014) Alternative assessment: Categorizations, supporting technologies and a model for betterment. Pensee J 76: 1.

4. Yan Y, Dittmann L (2014) Security challenges and solutions for telemedicine over EPON. IARIS 25: 263-240.

5. Saper N (2013) International cryptography regulation and the global information economy. J Tech Intel Prop 11: 673-688.

6. Ben-Jacob M (2016) Assessment: Classic and innovative approaches. J Soc Sci 5:46-51.

7. http://www.mobihealthnews.com/news/3-best-practices-telehealthinfrastructure

8. http://www.blackboard.com/

9. https://cft.vanderbilt.edu/guides-sub-pages/blooms-taxonomy/

10. http://www.iclicker.com/dnn/

11. https://www.skype.com/en

12. http://telehealth.org/sites/default/files/BroadbandandTelemedicine.pdf

13. Catherine P, Banta A, Trudy W (1999) Assessment essentials: planning implementing, and improving assessment in higher education. Ass Ess 1: 1-2

14. https://evisit.com/what-is-telemedicine/

15. Celia F, Fried B, Adam L (2003) Internet-mediated psychological services and the American Psychological Association Ethics Code. Psychotherapy 40: 103-111.

16. http://www.hipaajournal.com/hipaa-guidelines-on-telemedicine/

17. Weaver B (2013) Formal vs. informal assessments. 